\title{
Hydrochimie des eaux Souterraines de la Région du Poro (Côte d'Ivoire)
}

\author{
Adama Diabagaté, \\ Tié Albert Goula, \\ Gneneyougo Emile Soro, \\ Université NANGUI ABROGOUA d'Abidjan, Côte d'Ivoire, \\ Laboratoire de Géosciences et de l'Environnement
}

Doi: 10.19044/esj.2019.v15n9p247 URL:http://dx.doi.org/10.19044/esj.2019.v15n9p247

\section{Résumé}

Dans le but de caractériser les eaux souterraines de la région du Poro et d'appréhender les phénomènes à l'origine de leurs minéralisations, des analyses physicochimiques ont été effectuées sur 124 échantillons d'eau. L'analyse descriptive des paramètres physicochimiques a montré que les eaux sont acides et faiblement minéralisées dans l'ensemble. Les teneurs en manganèse et en fer sont parfois très élevées et au-dessus de la norme de potabilité OMS des eaux de consommation. Le diagramme de Piper a révélé que les eaux sont à environ 98\% hydrogénocarbonatées calciques et chlorurées sulfatées calciques à $2 \%$. L'Analyse en Composantes Principales (ACP) a mis en évidence deux grands phénomènes dans l'acquisition de la minéralisation des eaux. Le principal phénomène est la dissolution de la roche par le contact eau-roche et le second est lié aux apports d'eaux superficielles par infiltration. Dans l'ensemble, les eaux souterraines de la zone d'étude sont de bonne qualité. Très peu de nitrates y ont été détectés et les concentrations mesurées sont bien en-deçà de la directive pour l'eau potable. Les concentrations en manganèse et en fer dépassent parfois le critère esthétique et pourraient être une préoccupation pour la santé des enfants.

Mots-clés: Eaux souterraines, minéralisation, Qualité, Korhogo, Côte d'Ivoire 


\title{
Hydrochemicals of the Underground Waters of Poro Region (Ivory Coast)
}

\author{
Adama Diabagaté, \\ Tié Albert Goula, \\ Gneneyougo Emile Soro, \\ Université NANGUI ABROGOUA d'Abidjan, Côte d'Ivoire, \\ Laboratoire de Géosciences et de l'Environnement
}

\begin{abstract}
In order to characterize the groundwater of the Poro region and to apprehend the phenomena at the origin of their mineralization, physicochemical analyzes were carried out on 124 water samples. The descriptive analysis of physicochemical parameters showed that the waters are acidic and weakly mineralized overall. The levels of manganese and iron are sometimes very high and above the drinking water standard WHO drinking water. The Piper diagram revealed that the waters are approximately 98\% calcium carbonate and calcium sulphate chlorinated carbonate at $2 \%$. The Principal Component Analysis (PCA) has highlighted two major phenomena in the acquisition of mineralization of water. The main phenomenon is the dissolution of the rock by the water-rock contact and the second is related to the inflow of water by superficial infiltration. Overall, the groundwater in the study area is of good quality. Very few nitrates have been detected and the measured concentrations are well below the drinking water guideline. Concentrations of manganese and iron sometimes exceed the aesthetic criteria and could be a concern for the health of children.
\end{abstract}

Keywords: Groundwater, mineralization, Quality, Korhogo

\section{Introduction}

Pour pallier les problèmes d'Alimentation en Eau Potable (AEP) des populations, plusieurs projets sont initiés et orientés vers l'exploitation des ressources en eau disponibles en fonction des régions. Dans l'AEP des populations, les eaux souterraines ont l'avantage de fournir de l'eau de qualité car moins vulnérable à la pollution. Les activités anthropiques menaces de plus en plus la qualité physico chimique des eaux souterraines suites à l'infiltration de pollution d'origines diverses (OCDE, 2004 ; Abdelbaki et Boukli, 2007 ; Bricha et al., 2007 ; Koné et al., 2009 ;). En Côte d'Ivoire, 
plusieurs foyers de pollution ont été mise en exergue dans le bassin sédimentaire à travers différents travaux dont Soro et al., (2010); Ahoussi et al., (2012b) Selon Ismaïla et al, (2016). Les perturbations de la qualité physico-chimiques des eaux sont essentiellement le fait d'une agriculture extensive à usage incontrôlé d'intrants chimiques et d'une forte croissance démographique augmentant la pression sur les ressources en eau en particulier dans le bassin versant de la Comoé. Suscité pour être une alternative à l'alimentation en eau des populations de la région du Poro, la maitrise de la qualité physico-chimique des eaux souterraines de la région revêt un intérêt car le principe de prévention systématique est de loin préférable à celui qui consiste à traiter les eaux pour l'alimentation en eau potable lorsque leur qualité est détériorée (Mourey et Vernoux 2000). C'est donc pour soutenir cet avis, que cette étude est initiée pour caractériser la qualité physico-chimique des eaux souterraines de la région du Poro au regard de la directive OMS.

Pour réaliser cette étude, une campagne d'échantillonnage a été réalisée, au cours de laquelle les paramètres physico chimiques de 124 forages répartis dans la région du Poro ont été collectés et mesurés. Des méthodes d'analyse statistiques ont été utilisées pour le traitement des paramètres physico-chimiques.

\section{Échantillonnage et méthodes analytiques}

L'échantillonnage a été effectué sur 124 forages repartis dans la région du Poro. Les eaux des forages ont été recueillies après un pompage modéré à débit constant. La température $(\mathrm{T})$, le $\mathrm{pH}$, la conductivité électrique $(\mathrm{CE})$, et l'oxygène dissous ont été mesurés in situ à l'aide d'un multi paramètre de marque WWT 82 362. La turbidité a été également mesurée sur le terrain avec un turbidimètre de marque WTW $315 \mathrm{i}$ (de précision \pm 1 digit). Les coordonnées géographiques ont été obtenues à l'aide d'un GPS. Le réseau des points d'échantillonnage est présenté à la figure 1. Les échantillons d'eau ont été conservés à $4^{\circ}$ dans une glacière pendant le transport au laboratoire. Les paramètres chimiques, notamment les ions $\mathrm{HCO}_{3}{ }^{-}, \mathrm{Ca}^{2+}, \mathrm{Mg}^{2+}$ et $\mathrm{Cl}^{-}$ont été dosés par la méthode de titration avec l'acide chlorhydrique $0,1 \mathrm{~N}$ à partir d'un Titrateur digital (HACH 1690001). Quant aux autres ions $\mathrm{NO}_{3}{ }^{-}, \mathrm{SO}_{4}{ }^{2-}$, $\mathrm{Fe}^{2+}, \mathrm{K}^{+}, \mathrm{Na}^{+}, \mathrm{Mn}^{2+}, \mathrm{PO}_{4}{ }^{3-}$ et la $\mathrm{SiO}_{2}$, ils ont été dosés par la méthode des tests cuves du DR 6000. 


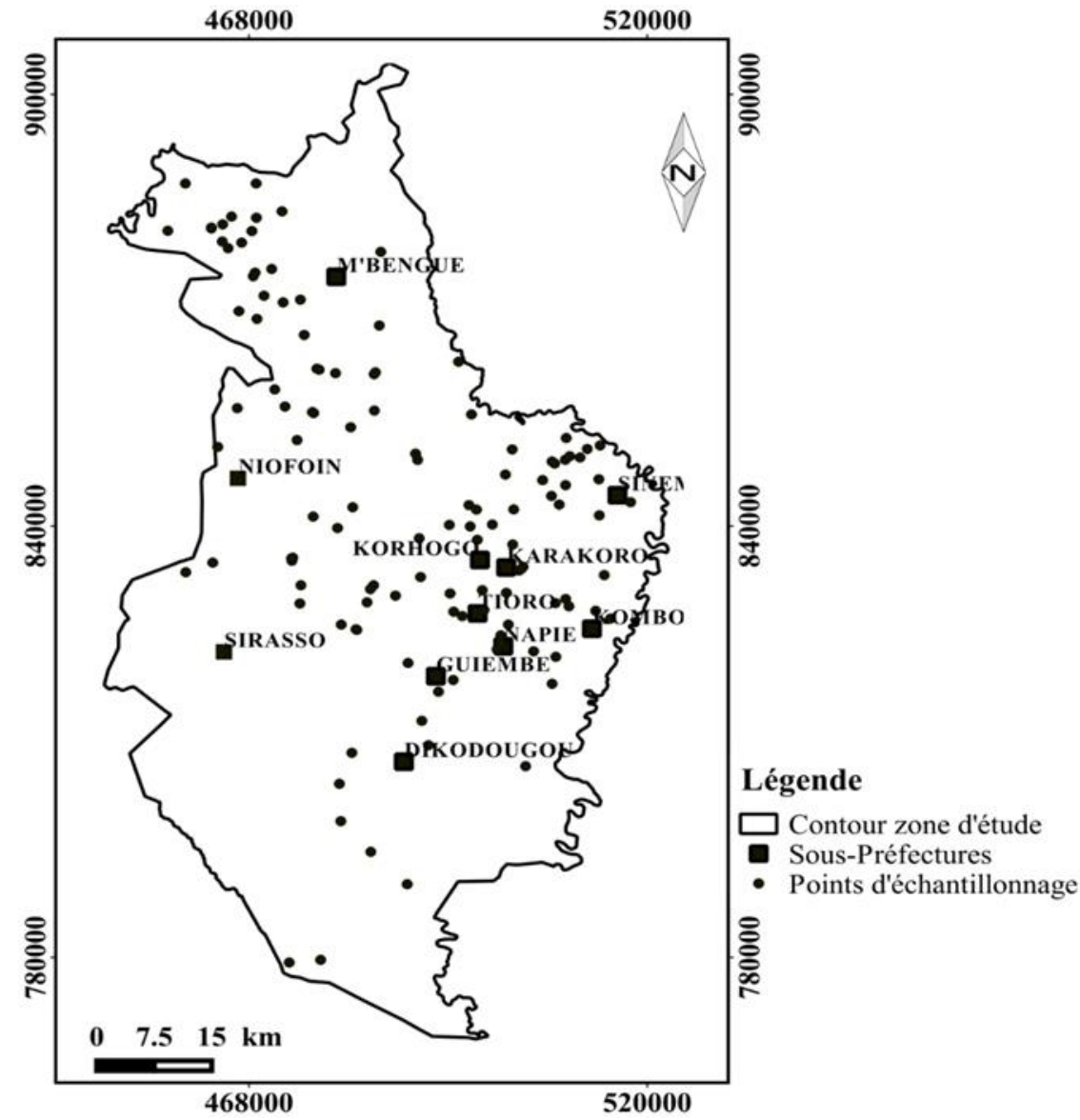

Figure 1 : Répartition des points d'échantillonnage dans la région du Poro

\section{Analyse hydro-chimique des données et Détermination des hydrofaciès}

La détermination des hydro-faciès a été fait à partir du logiciel diagrammes de piper (1953) version 2.00. Le diagramme de Piper (Figure 2) permet de représenter le faciès chimique d'un ensemble d'échantillons d'eau. Il est composé de deux triangles permettant de représenter le faciès cationique et le faciès anionique et d'un losange synthétisant le faciès global. Ce dernier est subdivisé en quatre losanges matérialisant un type d'eau (Orou et al., 2016). 


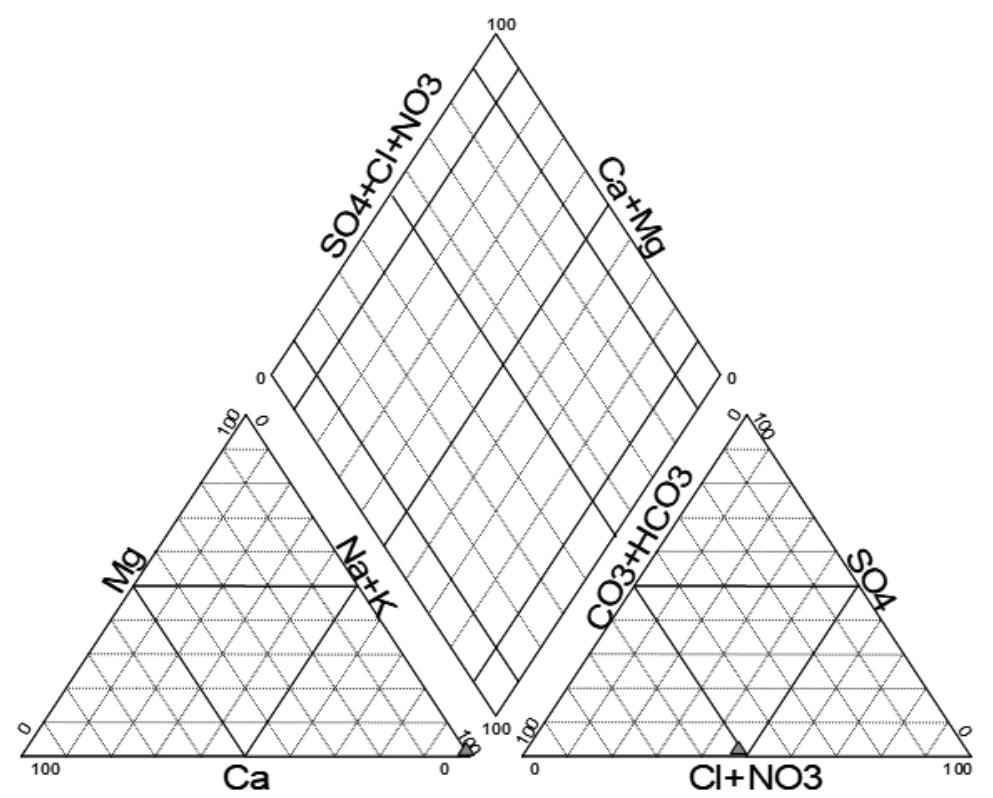

Figure 2 : Diagramme de Piper

\section{Analyse statistique des données}

L'analyse des données physico-chimiques est basée sur la statistique descriptive et l'analyse en composantes principales (ACP). L'analyse en composante principale ( $\mathrm{ACP})$ sert à mettre en évidence des similarités ou des oppositions entre variables et à repérer les variables les plus corrélées entre elles. La contribution de chaque variable dans la formation d'une composante principale est donnée par le coefficient de corrélation entre la variable et l'axe considéré. Plus le coefficient est important, plus la variable contribue à la formation de l'axe. Elle étudie les phénomènes qui sont à l'origine de la minéralisation des eaux. Les tests de corrélation de Pearson ont été réalisés à un niveau de significativité de $95 \%(\mathrm{p}<0,05)$ avec le logiciel STATISTICA7.1.

\section{Résultats}

\section{Paramètres physiques}

Les résultats des paramètres physico-chimiques mesurés in situ sont reportés dans le tableau 1 . La température moyenne enregistrée dans les eaux souterraines est de $26,89 \pm 0,41^{\circ} \mathrm{C}$ avec une température maximale de $28^{\circ} \mathrm{C}$. Les valeurs extrêmes du pH sont de 5,89 à Wolo dans la sous-préfecture de Sinématiali et 6,95 à Oléo dans la sous-préfecture de Korhogo avec une moyenne de 6,51 $\pm 0,18$ pratiquement similaire dans toutes les eaux.

Les eaux dans leur ensemble présentent une turbidité acceptable avec une moyenne de $0,51 \pm 0,21 \mathrm{NTU}$. Les valeurs de turbidité supérieure à $1 \mathrm{NTU}$ sont observées en F9, F36, F51, F75 respectivement dans les localités de 
Kadihoa dans la sous-préfecture de Dikodougou $(1,32)$; Solénandjeli et Wagniningavogo dans la sous-préfecture de Korhogo $(1,09)$ et à Noufou dans la sous-préfecture de M'bengué $(1,06)$. Tout comme la turbidité, la coloration a une évolution concomitante avec la turbidité. Il s'agit de F9, F36, F41, F51 et F75 dont les teneurs sont supérieures à 15 UCV. La conductivité électrique des eaux varie entre 73 et $674 \mu \mathrm{S} . \mathrm{cm}^{-1}$ avec une moyenne de 197,71 1112,14 $\mu \mathrm{S} . \mathrm{cm}^{-1}$. Les conductivités maximales de l'ensemble des eaux étudiées sont observées à Sonzorisso Dioula et Marah dans la sous-préfecture de Dikodougou avec des concentrations respectives de 753 et $674 \mu \mathrm{S} . c m^{-1}$. La majorité des échantillons d'eau a une conductivité électrique comprise entre $50 \mu \mathrm{S} . \mathrm{cm}^{-1}$ et $500 \mu \mathrm{S} . \mathrm{cm}^{-1}$ soit $98 \%$ des eaux correspondant à la classe des eaux faiblement minéralisées.

Tableau 1 : Statistique des paramètres chimiques des eaux souterraines

\begin{tabular}{|c|c|c|c|c|c|c|c|}
\hline \multicolumn{3}{|c|}{ Paramètres } & \multirow{2}{*}{$\begin{array}{r}\text { Moy } \pm \boldsymbol{\alpha} \\
26,89 \pm 0,41\end{array}$} & \multirow{2}{*}{$\begin{array}{l}\text { Min } \\
25,9\end{array}$} & \multirow{2}{*}{$\begin{array}{l}\text { Max } \\
28\end{array}$} & \multirow{2}{*}{$\begin{array}{l}\text { Variance } \\
0,17\end{array}$} & \multirow{2}{*}{$\begin{array}{r}\begin{array}{r}\text { OMS } \\
(\mathbf{2 0 1 1})\end{array} \\
25-30\end{array}$} \\
\hline \multirow{5}{*}{ 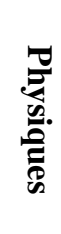 } & $\mathbf{T}$ & ${ }^{\circ} \mathbf{C}$ & & & & & \\
\hline & PH & $(-)$ & $6,51 \pm 0,18$ & 5,89 & 6,95 & 0,03 & $6,5-8,5$ \\
\hline & Turb & UNT & $0,51 \pm 0,21$ & 0,3 & 1,32 & 0,05 & - \\
\hline & Coul & $\mathbf{U C V}$ & $10,33 \pm 5,48$ & 4 & 30 & 30,06 & $-<15$ \\
\hline & $\mathbf{C E}$ & $\mu \mathrm{S} . \mathrm{cm}^{-1}$ & $197,71 \pm 112,14$ & 73 & 674 & 12576,22 & $500-1500$ \\
\hline \multirow{15}{*}{ 串. } & $\mathrm{Na}^{+}$ & mg. $\mathbf{L}^{-1}$ & $0,014 \pm 0,04$ & 0,01 & 0,45 & 0,00 & - \\
\hline & TH & ${ }^{\circ} \mathbf{F}$. & $7,17 \pm 5,28$ & 1,6 & 25,6 & 27,89 & 30 \\
\hline & $\mathrm{HCO}_{3}^{-}$ & mg. $L^{-1}$ & $121,73 \pm 61,65$ & 42,7 & 359,9 & 3800,07 & - \\
\hline & $\mathrm{Ca}^{2+}$ & mg. $\mathrm{L}^{-1}$ & $21,58 \pm 16,24$ & 2,825 & 78,56 & 263,92 & - \\
\hline & $\mathrm{Mg}^{2+}$ & mg. $\mathrm{L}^{-1}$ & $3,84 \pm 2,94$ & 0,02 & 14,56 & 8,63 & - \\
\hline & $\mathbf{K}^{+}$ & mg. $\mathrm{L}^{-1}$ & $0,02 \pm 0,094$ & 0,01 & 1 & 0,01 & $<5$ \\
\hline & $\mathrm{Fe}^{2+}$ & mg. $\mathrm{L}^{-1}$ & 0,134 & 0,01 & 0,42 & 0,02 & $<0,3$ \\
\hline & $\mathrm{Mn}^{2+}$ & mg. $\mathrm{L}^{-1}$ & $0,04 \pm 0,054$ & 0,01 & 0,25 & 0,00 & $<0,05$ \\
\hline & $\mathbf{O H}^{-}$ & mg..$^{-1}$ & $0,17 \pm 1,79$ & 0,01 & 20 & 3,22 & - \\
\hline & $\mathrm{NH}_{4}{ }^{+}$ & mg. $\mathrm{L}^{-1}$ & $0,05 \pm 0,46$ & 0,01 & 5,2 & 0,22 & 1,5 \\
\hline & $\mathrm{NO}_{3}^{-}$ & mg..$^{-1}$ & $1,44 \pm 4,35$ & 0,01 & 25 & 18,88 & $\geq 50$ \\
\hline & $\mathrm{NO}_{2}^{-}$ & mg..$^{-1}$ & $0,016 \pm 0,032$ & 0,01 & 0,3 & 0,00 & 0,1 \\
\hline & $\mathrm{Cl}^{-}$ & mg. $L^{-1}$ & $7,92 \pm 8,12$ & 3,546 & 49,64 & 65,86 & $\leq 250$ \\
\hline & $\mathrm{SO}_{4}{ }^{2-}$ & mg. $\mathrm{L}^{-1}$ & $1,34 \pm 10,47$ & 0,01 & 90 & 109,77 & $\leq 250$ \\
\hline & $\mathrm{PO}_{4}{ }^{3-}$ & mg. $\mathrm{L}^{-1}$ & $0,09 \pm 0,07$ & 0,01 & 0,38 & 0,01 & - \\
\hline
\end{tabular}

Les valeurs du coefficient de variation $(\mathrm{CV})$ des paramètres physiques sont très faibles $(0,05$ à 0,17$)$ pour la température, le $\mathrm{pH}$ et la turbidité alors qu'elles sont élevées pour la conductivité électrique et la couleur. Ces derniers ont des coefficients de variation $(>1)$. Ces résultats montrent que la température, le $\mathrm{pH}$ et la turbidité sont très homogènes donc varient très peu 
d'un point à un autre alors que la conductivité électrique est hétérogène donc varie beaucoup dans l'espace.

\section{Analyses des paramètres chimiques}

Les valeurs moyennes des nitrates $\left(\mathrm{NO}_{3}{ }^{-}\right)$, des nitrites $\left(\mathrm{NO}_{2}{ }^{-}\right)$, des chlorures $\left(\mathrm{Cl}^{-}\right)$, du sulfate $\left(\mathrm{SO}_{4}{ }^{2-}\right)$ et du phosphate $\left(\mathrm{PO}_{4}{ }^{3-}\right)$ sont respectivement de $1,44 \pm 4,35 ; 0,016 \pm 0,032 ; 7,92 \pm 8,12 ; 1,34 \pm 10,47$ et $0,09 \pm 0,07 \mathrm{mg}$. L 1 . Comme on peut le constater sur la figure 3 , les concentrations de ces anions sont variables d'un site à un autre. Ainsi les forages F3 (25 mg.L $\left.{ }^{-1}\right)$, F7 (16 mg. $\left.\mathrm{L}^{-1}\right)$, F10 (20 mg. $\left.\mathrm{L}^{-1}\right)$ et F79 (20 mg. $\left.\mathrm{L}^{-1}\right)$ ont des teneurs en nitrate supérieures à $10 \mathrm{mg} \cdot \mathrm{L}^{-1}$. Les concentrations en sulfate les plus significatives sont observées en F33 (90 mg. $\left.\mathrm{L}^{-1}\right)$ et F109 (75 mg. $\left.\mathrm{L}^{-1}\right)$. La concentration moyenne de $\mathrm{Cl}^{-}$est de 7,92 $\pm 8,12 \mathrm{mg} \cdot \mathrm{L}^{-1}$. Cependant, des concentrations significatives de chlorure dans les eaux souterraines bien qu'en dessous de la recommandation OMS (2011) sont observées dans les localités de Dékélévogo dans la sous-préfecture de M'bengué $\left(42,55 \mathrm{mg} . \mathrm{L}^{-1}\right)$ et Sonzorisso Dioula dans la sous-préfecture de Dikodougou $\left(49,64 \mathrm{mg} . \mathrm{L}^{-1}\right)$ précisément en F65 et F7.

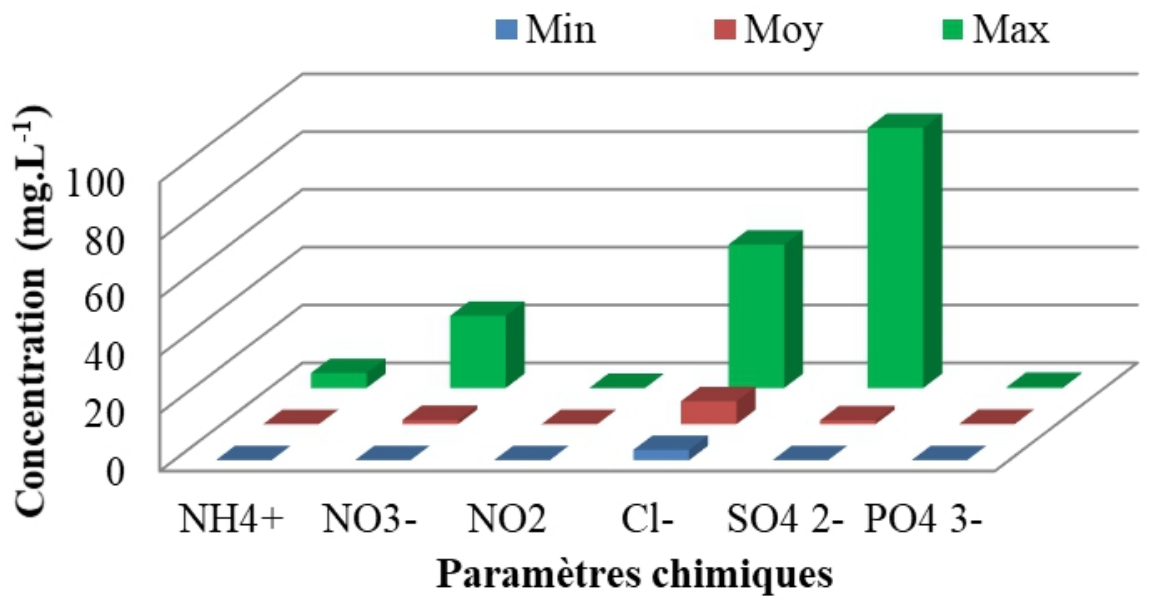

Figure 3 : Concentration des anions majeurs dans les sites de prélèvement des eaux

Les valeurs minimales et maximales de la dureté sont respectivement de $1,6{ }^{\circ} \mathrm{F}$ et $25,6{ }^{\circ} \mathrm{F}$ avec une moyenne de $7,17 \pm 5,28^{\circ} \mathrm{F}$. Quant aux concentrations du calcium $\left(\mathrm{Ca}^{2+}\right)$ et l'hydrogénocarbonate $\left(\mathrm{HCO}_{3}{ }^{-}\right)$, elles sont abondantes dans les eaux échantillonnées. La valeur maximale en $\mathrm{Ca}^{2+}$ est de 78,56 mg. $\mathrm{L}^{-1}$ et celui de $\mathrm{HCO}_{3}{ }^{-}$est de 359,9 mg.L ${ }^{-1}$. Ces deux paramètres ont des coefficients de variation supérieure à $1(>1)$. Ces deux paramètres sont hétérogènes donc varient beaucoup dans l'espace. Ils sont à l'origine du faciès hydrogénocarbonate calcique. Les eaux souterraines de la zone d'étude sont donc douces. Il ressort de la figure 4 que les ions $\mathrm{Fe}^{2+}$ et $\mathrm{Mn}^{2+}$ enregistrent dans les eaux souterraines des concentrations variant respectivement de 0 à 
0,42 mg. $\mathrm{L}^{-1}$ et de 0 à $0,25 \mathrm{mg} . \mathrm{L}^{-1}$. Les eaux échantillonnées présentent $29,03 \%$ de teneurs en manganèse supérieures à la directive OMS (2011) et 22,58\% de $\mathrm{Fe}^{2+}$.

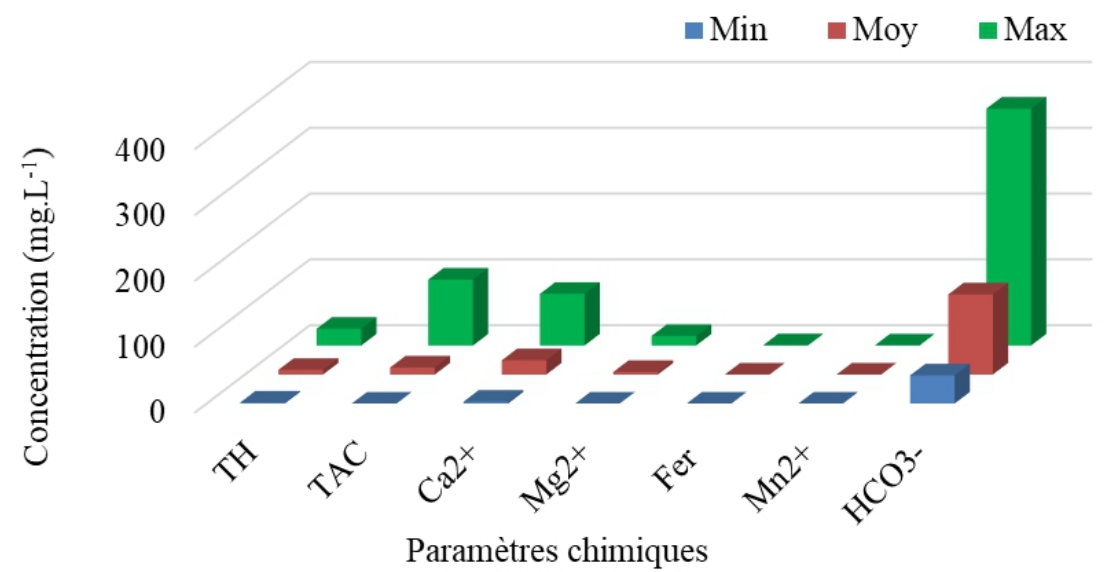

Figure 4 : Évolution des concentrations en ion des eaux étudiées.

\section{Classification hydrochimique des eaux souterraines}

Les analyses exprimées en $\mathrm{mg} / \mathrm{l}$ des eaux souterraines ont été reportées sur les diagrammes de Piper (Figure 5). Ceux-ci représentent la part respective exprimée en pourcentage d'éléments majeurs contenus dans l'eau. Chaque point du diagramme est représentatif d'un point d'eau de forage. Dans ce diagramme, on remarque que dans le triangle des cations, sept (7) points s'approchent du pôle calcique magnésien, alors que sur le plan des anions ils tendent vers le pôle des hydrogénocarbonates chloriques. Le faciès hydrogénocarbonate calcique domine les eaux souterraines de la région (soit $94 \%$ ). Le faciès chloruré sulfaté calcique ne présente que $6 \%$ des échantillons d'eau de la région. 


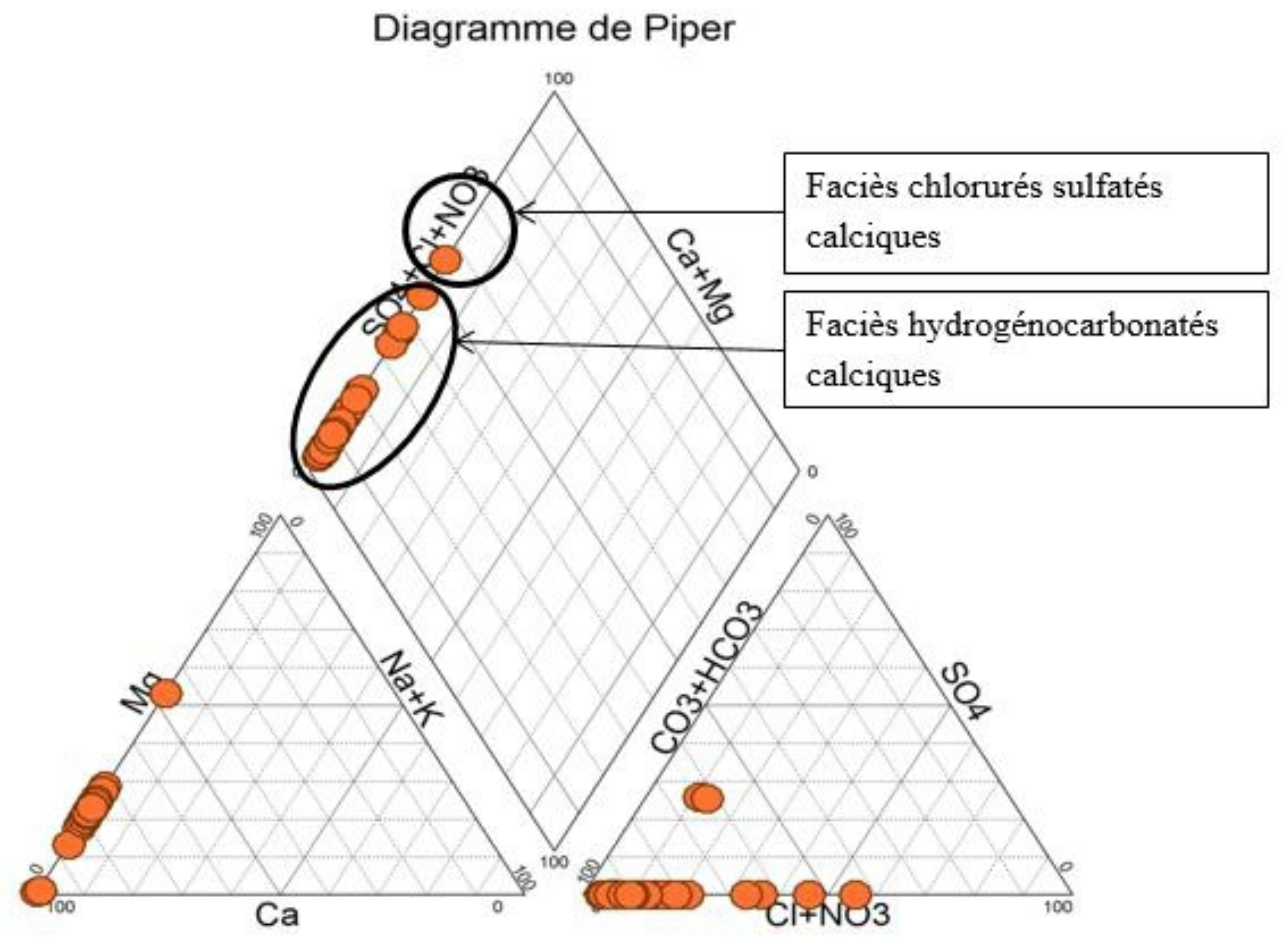

Figure 5. Diagramme de Piper des eaux de forages de la région du Poro

Deux (2) grandes familles d'eau se distinguent dans les eaux souterraines de la région : Les eaux à faciès chlorurés sulfatés calciques et les eaux à faciès hydrogénocarbonatés calciques qui sont les plus fréquentes.

\section{Analyse statistique}

Les valeurs propres qui permettent d'apprécier la variance exprimée par chaque facteur et de montrer l'importance du caractère représenté par l'axe factoriel par rapport aux autres sont consignées dans le tableau 2. Le pourcentage de variances exprimées est de $31,3 \%$ pour le facteur F1 ; 17,56\% pour le facteur F2 ; 5,79\% pour le facteur 3 et de 5,64\% pour le facteur 4 . La représentation à l'aide des deux premiers facteurs rend compte de manière satisfaisante de la structure des nuages de points.

Tableau 2: Valeurs propres et pourcentages exprimés par les axes principaux

\begin{tabular}{lllll}
\hline & F1 & F2 & F3 & F4 \\
\hline Valeur Propre & 6,88 & 3,86 & 1,27 & 1,24 \\
\% de Variance exprimée & 31,3 & 17,56 & 5,79 & 5,64 \\
Cumul de valeur propre & 6,8 & 10,75 & 12,02 & 13,27 \\
\% de Variance exprimée cumulée & 31,3 & 48,86 & 54,66 & 60,31 \\
\hline
\end{tabular}




\section{Corrélation entre les variables}

La représentation à l'aide des deux premiers facteurs rend compte de manière satisfaisante de la structure des nuages de points. La liaison existante entre toutes les variables prises deux à deux et les coefficients de corrélation entre ces différentes variables sont donnés dans le tableau 3.

Tableau 3 : Matrice de corrélation entre les variables

\begin{tabular}{lllllllllllll}
\hline & $\mathbf{C E}$ & $\mathbf{P H}$ & $\mathbf{T u r b}$ & $\mathbf{N H}_{4}^{+}$ & $\mathbf{C a}^{\mathbf{2 +}}$ & $\mathbf{M g}^{\mathbf{2 +}}$ & $\mathbf{F}^{2+}$ & $\mathbf{M n}^{\mathbf{2 +}}$ & $\mathbf{N O}^{-}$ & $\mathbf{C l}^{-}$ & $\mathbf{P O}_{4}{ }^{\mathbf{3}^{-}}$ & $\mathbf{H C O}^{-}$ \\
\hline $\mathbf{C E}$ & 1,00 & & & & & & & & & & & \\
$\mathbf{P H}$ & $\mathbf{0 , 6 5}$ & 1,00 & & & & & & & & & & \\
Turb & 0,02 & $-0,35$ & 1,00 & & & & & & & & & \\
$\mathbf{N H}_{4}{ }^{+}$ & $-0,08$ & $-0,31$ & $-0,04$ & 1,00 & & & & & & & & \\
$\mathbf{C a}^{2+}$ & $\mathbf{0 , 9 2}$ & $\mathbf{0 , 6 2}$ & 0,01 & $-0,09$ & 1,00 & & & & & & & \\
$\mathbf{M g}^{2+}$ & $\mathbf{0 , 8 5}$ & $\mathbf{0 , 5 7}$ & $-0,03$ & $-0,11$ & 0,91 & 1,00 & & & & & & \\
$\mathbf{F}^{2+}$ & 0,02 & $-0,35$ & $\mathbf{0 , 7 6}$ & $-0,10$ & 0,06 & 0,07 & 1,00 & & & & & \\
$\mathbf{M n}^{2+}$ & $-0,01$ & $-0,36$ & $\mathbf{0 , 7 2}$ & $-0,06$ & 0,03 & 0,06 & $\mathbf{0 , 8 8}$ & 1,00 & & & & \\
$\mathbf{N O}_{3}{ }^{-}$ & $\mathbf{0 , 6 8}$ & 0,32 & 0,14 & $-0,03$ & $\mathbf{0 , 6 1}$ & $\mathbf{0 , 6 4}$ & 0,16 & 0,13 & 1,00 & & & \\
$\mathbf{C l}^{-}$ & $\mathbf{0 , 9 0}$ & $\mathbf{0 , 5 7}$ & 0,03 & $-0,04$ & $\mathbf{0 , 8 6}$ & $\mathbf{0 , 7 0}$ & $-0,00$ & $-0,03$ & $\mathbf{0 , 5 8}$ & 1,00 & & \\
$\mathbf{P O}_{4}{ }^{3-}$ & $\mathbf{0 , 7 3}$ & 0,42 & 0,08 & 0,14 & $\mathbf{0 , 7 2}$ & $\mathbf{0 , 7 0}$ & 0,05 & 0,04 & $\mathbf{0 , 6 0}$ & $\mathbf{0 , 6 5}$ & 1,00 & \\
$\mathbf{H C O}_{3}{ }^{-}$ & $\mathbf{0 , 6 9}$ & 0,50 & 0,04 & $-0,02$ & $\mathbf{0 , 7 8}$ & $\mathbf{0 , 7 8}$ & 0,08 & 0,06 & 0,29 & $\mathbf{0 , 6 3}$ & $\mathbf{0 , 6 5}$ & 1,00 \\
\hline
\end{tabular}

L'analyse du tableau révèle des corrélations faibles et négatives, faibles, fortes et positives entre certaines variables. Ainsi, la CE présente de très fortes corrélations avec $\mathrm{Ca}^{2+}(0,92), \mathrm{Cl}^{-}(0,90), \mathrm{Mg}^{2+}(0,85)$, et une corrélation moyenne avec $\mathrm{HCO}_{3}(0,69), \mathrm{NO}_{3}^{-}(0,68), \mathrm{PO}_{4}{ }^{3-}(0,73)$. Il existe de fortes corrélations entre Turbidité et $\mathrm{Fe}^{2+}(0,76), \mathrm{Mn}^{2+}(0,72)$. Ces deux derniers paramètres sont fortement corrélés $(0,88)$. Les corrélations sont également importantes entre $\mathrm{Ca}^{2+}$ et $\mathrm{Cl}^{-}(0,86), \mathrm{HCO}^{3}(0,78), \mathrm{PO}_{4}{ }^{3-}(0,72)$, mais moyenne corrélé avec $\mathrm{NO}_{3}{ }^{-}(0,61)$. Quant au $\mathrm{Mg}^{2+}$, il présente les mêmes caractéristiques que $\mathrm{Ca}^{2+}$ avec des valeurs respectives $(0,78 ; 0,70 ; 0,70 ; 0,64)$. Par ailleurs, ces deux paramètres sont moyennement corrélés au $\mathrm{pH}$. ${\mathrm{Le} \mathrm{NO}_{3}}^{-}$ $(0,47)$, le $\mathrm{Cl}^{-}$et le $\mathrm{PO}_{4}{ }^{3-}$ sont moyennement corrélés entre eux. Respectivement entre $\mathrm{NO}_{3}{ }^{-}$et $\mathrm{Cl}^{-}(0,58), \mathrm{PO}_{4}{ }^{3-}(0,60)$; entre $\mathrm{Cl}^{-}$et $\mathrm{PO}_{4}{ }^{3-}(0,65), \mathrm{HCO}_{3}{ }^{-}(0,63)$. Les corrélations moyennes s'observent entre le $\mathrm{pH}$ et $\mathrm{CE}(0,65)$. Il existe également de très faibles corrélations négatives mais pas significatives.

\section{Origine des ions dans les eaux souterraines}

\section{Analyse des variables dans les plans factoriels}

Le plan factoriel (F1-F2) représente à lui seul 37,05\% des variances exprimées donc des informations recherchées. L'analyse des variables de l'ACP dans le plan factoriel F1-F2 est présentée par les figures 5 et 6 . Ces graphes mettent en évidence trois grands regroupements des paramètres étudiés dans les eaux souterraines. 
Le premier groupe, est commandé dans sa partie négative du facteur F1 par les variables que sont : $\mathrm{pH}, \mathrm{CE}, \mathrm{Cl}^{-}, \mathrm{SO}_{4}{ }^{2-} \mathrm{HCO}_{3}{ }^{-} ; \mathrm{OH}^{-}, \mathrm{TAC}, \mathrm{Ca}^{2+}$ et $\mathrm{Mg}^{2+}, \mathrm{NO}_{3}{ }^{-}$.

Le facteur 2 exprime à lui seul 17,5\% de l'inertie du nuage des points représentatifs des ouvrages et est déterminé par $\mathrm{T}^{\circ} \mathrm{C}, \mathrm{Na}^{+}, \mathrm{K}^{+}, \mathrm{NH}_{4}^{+}, \mathrm{NO}_{2}{ }^{-}$. Le troisième groupe composé de fer, du manganèse et de couleur est contrôlé dans sa partie négative du plan factoriel F2.

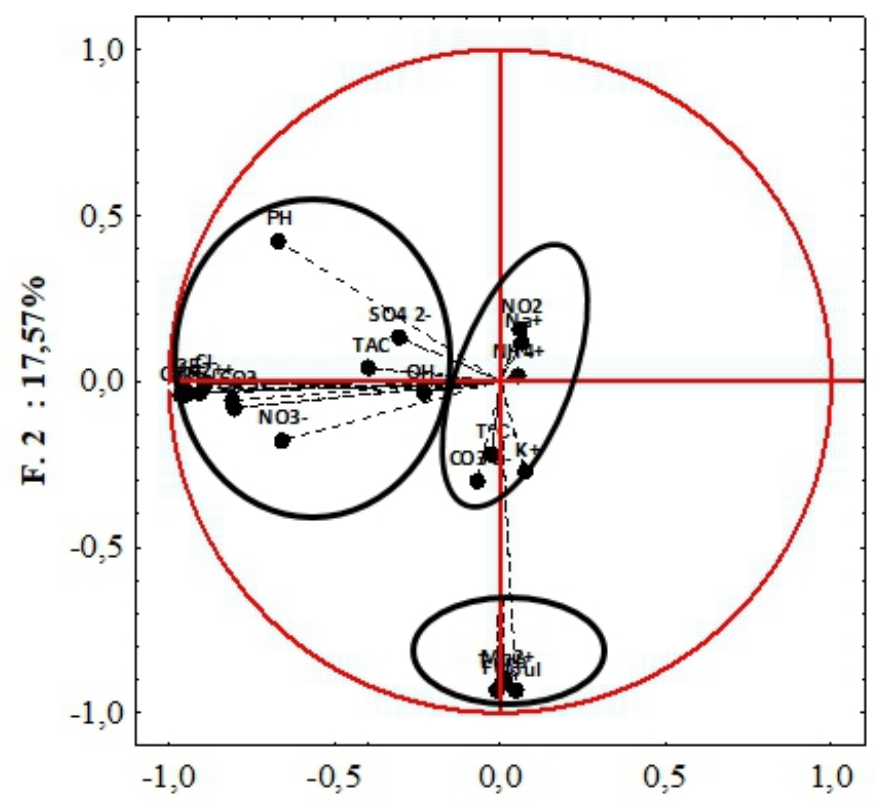

F. $1: 31,30 \%$

Figure 5 : Espace des variables du plan factoriel F1xF2

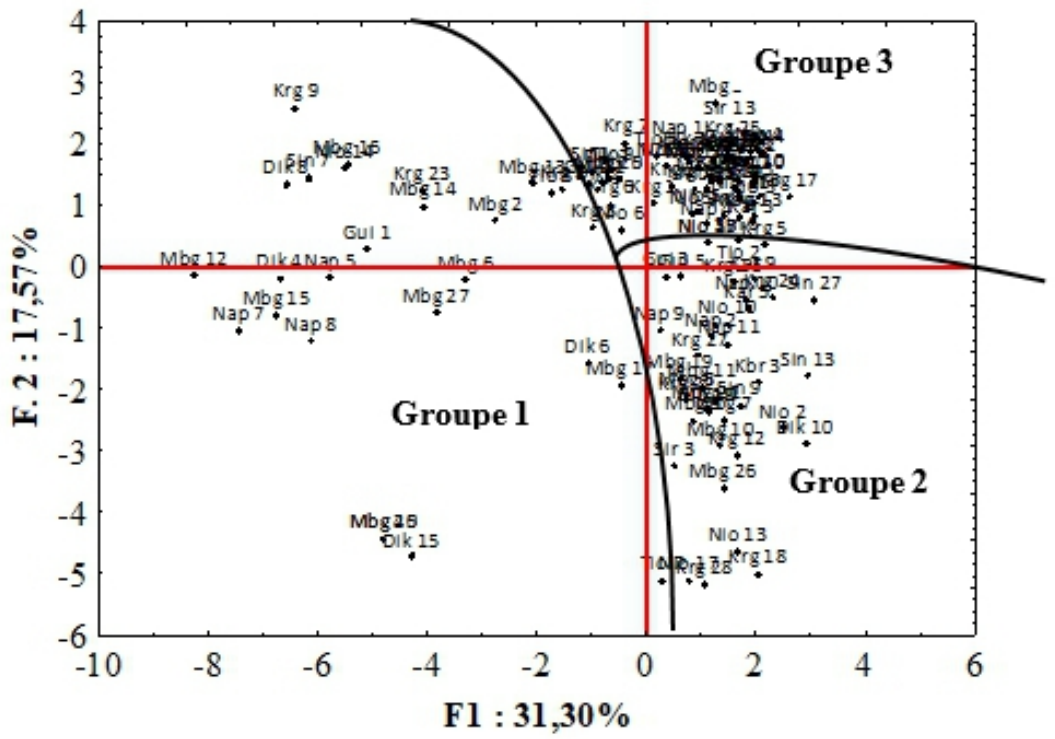

Figure 6 : Espace des individus du plan factoriel $\mathrm{F} 1 \times \mathrm{F} 2$ 


\section{Discussion}

Les résultats des analyses physico-chimiques des eaux souterraines de la région du Poro montrent que les eaux sont légèrement acides avec un $\mathrm{pH}$ moyen de 6,9 $\pm 1,16$. L'acidité des eaux souterraines selon Ahoussi et al. (2012) constitue l'un des traits caractéristiques des eaux souterraines de Cote d'Ivoire. En effet, en zone tropicale humide, cette acidité proviendrait principalement de la décomposition de la matière organique végétale, avec la production de $\mathrm{CO}_{2}$ dans les premières couches du sol Oga et al. (2009), Lasm et al. (2011), Kouassi et al. (2012). La prédominance des ions hydrogénocarbonates $\left(\mathrm{HCO}_{3}{ }^{-}\right)$qui s'associe au cation $\mathrm{Ca}^{2+}$ dans les eaux échantillonnées serait une conséquence de l'attaque acide des roches. Elle constitue par ailleurs une caractéristique des eaux souterraines des régions de socle de Côte d'Ivoire et d'Afrique (Biémi, 1992; Soro 2002). Ces deux éléments sont à l'origine du faciès hydrogénocarbonaté calcique des échantillons d'eau.

Les eaux étudiées sont faiblement minéralisées $(197,71 \pm 112,14$ $\mu \mathrm{S} . \mathrm{cm}^{-1}$ ) avec une minéralisation assez élevée à Marah dans la sous-préfecture de Dikodougou) $\left(674 \mu \mathrm{S} . \mathrm{cm}^{-1}\right)$. Cette faible minéralisation des eaux selon Youan Ta et al. (2015), pourrait s'expliquer par le caractère très peu soluble des roches encaissantes. Les températures de ces eaux au-dessus de $25^{\circ} \mathrm{C}$, ne constituent pas un danger pour le consommateur. Cependant, les fortes teneurs en fer et en manganèse respectivement $21,77 \%$ et $29,03 \%$ enregistrées dans certaines localités constituent un problème majeur d'acceptabilité pour les populations. En effet, ces deux éléments confèrent à l'eau un goût métallique désagréable et une coloration rougeâtre qui pourrait être relative à une désoxygénation de l'eau par l'activité organique dans le sol et dans la zone non saturée (Goné, 2005). Par ailleurs, les teneurs en fer et en manganèse élevées dans certaines localités, pourrait être attribuées aux formations géologiques de la région comme l'ont signalé Ahoussi et al. (2013) Youan Ta et al. (2015) dans l'Ouest montagneux et à l'Est de la Cote d'Ivoire.

Le mécanisme de l'acquisition de la minéralisation des eaux a été mis en évidence par l'ACP. Le facteur F1 définit donc l'axe de minéralisation globale des eaux. Cependant, les regroupements de part et d'autre de cet axe

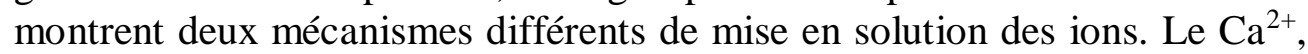
le $\mathrm{Mg}^{2+}$ et $\mathrm{TH}$ sont issus de l'altération des roches suite à un long temps de contact eau-roche et de l'hydrolyse acide des minéraux silicatés (Rodier,

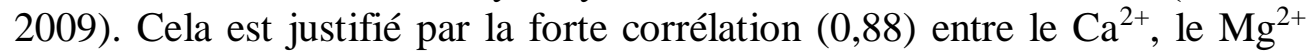
d'une part et d'autre part, par la corrélation moyenne entre le $\mathrm{pH}$ avec $\mathrm{CE}$ $(0,65), \mathrm{Mg}^{2+}(0,85)$ et $\mathrm{Ca}^{2+}(0,92)$.

Un autre mécanisme intervenant dans la minéralisation des eaux est régi par les apports d'eaux superficielles des surfaces agricoles et l'intrusion des eaux usées domestiques. Ce processus est mis en exergue par la corrélation 
entre $\mathrm{pH}$ et $\mathrm{NO}_{3}{ }^{-}(0,68)$ et $\mathrm{Cl}^{-}(0,90), \mathrm{PO}_{4}{ }^{3-}(0,73)$. La présence de ces éléments dans une eau souterraine dénote de l'influence des apports anthropiques sur la qualité des eaux souterraines. Certains auteurs Béchir et al. (2007), Blé et al. (2016), Benaricha et al. (2017), ont établi un lien entre la dégradation des nappes et la pression anthropique. Selon eux, le rejet d'effluents domestiques et le lessivage des terres cultivées renfermant des engrais phosphatés ou traités par certains pesticides en sont les causes. La concentration en nitrates obtenue dans les eaux de Sonzorisso dioula dans la sous-préfecture de Dikodougou (F3) et les teneurs en phosphates soit près de 36,29\% de concentrations supérieures à $0,1 \mathrm{mg} . \mathrm{L}^{-1}$ justifie une pratique intense d'activités agricoles dans la zone.

Les eaux souterraines de la région sont généralement acides. Cet aspect peut conduire à la fois à une dégradation des matériaux à base de ciment et à une corrosion des conduites d'eau et des appareils métalliques. La teneur anormale de nitrates à Sonzorisso dioula peut occasionner la méthémoglobinémie chez le bébé et des troubles mentaux chez l'adulte. Les teneurs élevées en phosphates constituent selon Eblin et al. (2014) un indice de pollution. Les fortes teneurs en fer et à un degré moindre en manganèse ont des inconvénients sur la qualité organoleptique des eaux.

\section{Conclusion}

L'approche statistique géochimique a permis de caractériser les propriétés hydrogéochimiques et de comprendre les processus majeurs de la minéralisation des ressources en eau souterraine de la région du Poro dans le nord de la Côte d'Ivoire. Ces eaux sont acides et faiblement minéralisées, riches en $\mathrm{Fe}^{2+}$ et $\mathrm{Mn}^{2+}$. L'origine de la minéralisation est due à l'altération des roches encaissantes et à l'hydrolyse des minéraux silicatés et ferromagnésiens ainsi qu'une pollution anthropique. Ces résultats constituent une étape préliminaire dans la connaissance du fonctionnement hydrogéochimique et une base de suivi de la qualité physico-chimique des eaux dans la zone d'étude.

\section{Remerciements}

Les auteurs de cet article remercient l'Office National de l'Eau Potable (ONEP) et le bureau d'étude Arc Ingénierie pour leur avoir permis de collecter les données d'analyses physico-chimiques utilisées dans cette étude.

Ils remercient également les instructeurs dont les critiques et suggestions ont permis d'améliorer le présent article. 


\section{References:}

1. Abdelbaki C. et Boukli H. F. (2007): Etude du phénomène de dégradation des eaux souterraines du groupement urbain de Tlemcen. Revue des Energies Renouvelables, 10(2) : 257-263.

2. Ahoussi K., Youan T., Loko S., Adja M., Lasm T. et Jourda J. (2012) Étude hydrogéochimique des eaux des aquifères de fractures du socle Paléo-protérozoïque du Nord-Est de la Côte d'Ivoire : Cas de la région de Bondoukou. Afrique SCIENCE, 08(3) : 51- 68

3. Ahoussi K., Koffi Y. B., Kouassi A. M., Soro G., Biemi J. (2013). Étude hydrochimique et microbiologique des eaux de source de l'ouest montagneux, Cote d'Ivoire : Cas du village de Mangouin-Yrongouin (souspréfecture de Biankouman), J. Appl. Biosci, 63: 4703 - 4719

4. Biémi J. (1992) : Contribution à l'étude géologique, hydrogéologique et par télédétection des bassins versants subsahariens du socle précambrien d'Afrique de l'Ouest : hydro structurale, hydrodynamique, hydrochimie et isotopique des aquifères discontinusde sillons et des aires granitiques de la Haute Marahoué (Côte d'Ivoire), Thèse d'Etat, Univ. Cocody, 480p.

5. Blé L. O., Aké G. E., Soro T., Soro G., Kouadio E. Y. (2016). Evaluation de la Qualité Hydrochimique des Eaux Souterraines de la Région de Bonoua. (Sud-Est de la Côte d'Ivoire). International Journal of Innovation and Applied Studies. 14 (4) : 896-907

6. Béchir B.T., Khalifa R., Houda B. (2007) : Elimination de la turbidité par oxygénation et filtration successives des eaux de la station de Sfax (Sud de la Tunisie). Rev. Sci. Eau, 20 (4): 355-365.

7. Benaricha B, Khaldi A, Elouissi A, Mouassa S, Zaagane M. (2017). Geochemical characterization the waters of foggaras the continental intercalary aquifer of Timinoune region (South West Alegria). $J$ Fundam Appl Sci. 9(1): 51-73.

8. Bricha s., Ounine k., Oulkheir s., El haloui n., ttarassi b. (2007). Etude de la qualité physicochimique de la nappe phréatique M'nasra (Maroc). Afrique SC. 03 (3): 391-404.

9. Eblin S.G., Soro G.M., Sombo A (2014). Hydrochimie des eaux souterraines de la région d'adiaké (sud-est côtier de la côte d'ivoire). Larhys J., pp. 193-214

10. Goné DL, Savané I, Yao NA, Biémi J. 2005. Mobilité relative des cations majeurs lors de l'altération des roches et acquisition de la minéralisation des eaux souterraines dans le degré carré de Man. Sci. Nat., 2(1) : 85-94.

11. Ouattara I., Kamagate B., Dao A., Noufé D., Savane I. (2016). Processus de minéralisation des eaux souterraines et transfert de flux en milieu de socle fissure: cas du bassin versant transfrontalier de la 
Comoe (Cote d'Ivoire, Burkina Faso, Ghana), Int. J. Innov. Applied Studies, 17(1):57-69

12. Kone M., Bonou 1., Bouvet y., Joly p., Koulidiaty j. (2009). Etude de la pollution des eaux par les intrants agricoles : cas de cinq zones d'agriculture intensive du Burkina Faso. Sud Sci. et Techno., 17 :5-15.

13. Kouassi M. A., Ahoussi E. K., Koffi B.Y., Ake Y. A. Biemi J. (2012). Caractérisation hydrogéochimique des eaux des aquifères fissurés de la zone Guiglo-Duekoué (Ouest de la Côte d'Ivoire) Int. J. Biol. Chem. Sci. 6(1): 504-518.

14. Lasm T, ASM, Lasme O, Oga MS, Youanta M, Baka D, Kouamé KF, Yao KT. (2011). Caractérisation hydrochimique des aquifères fissurés de la région de SanPedro (Sud-Ouest de la Côte d'Ivoire). International Journal of Biological and Chemical Sciences, 5(2): 642-662.

15. Mourey v., vernoux J. (2000) : Les risques pesant sur les nappes d'eau souterraine d'Ile-de-France. Annales des mines. Pp : 32-35

16. Oga M., Lasm T., Yao T., Soro N., Saley M., Kouassi D., et Gnamba F., (2009) Caractérisation chimique des eaux des aquifères de Fracture : Cas de La Région de Tiassalé en Côte d'Ivoire. European Journal of Scientific Research, 31(1): 72 - 87

17. Ohou M.J.A. (2009). Impacts des activités anthropiques sur la qualité physico-chimique des ressources en eau souterraine du bassin versant de la Lobo (Buyo, Sudouest de la Côte d'Ivoire) : Cas du nitrate. Thèse de Doctorat, UFR des Sciences et Gestion de l'Environnement, Université d'Abobo-Adjamé (Abidjan, Côte d'Ivoire), $118 \mathrm{p}$

18. Orou R. K. Soro G. Soro D.T. Fossou R.M. Onetie O. Z. Ahoussi E. K. Soro N. (2016). Variation Saisonnière De La Qualité Physico Chimique Des Eaux Souterraines Des Aquifères d'Altérites Du Département d'Agboville (Sud-Est De La Côte d'Ivoire) Eur. Sc. J., 12(17) :213-240.

19. O.M.S. 2011. Guidelines for drinking-water quality. 4th ed. 564p.

20. OCDE (2004). Agriculture et environnement : Enseignement tirés de dix ans (1993-2003) des travaux de l'OCDE, 40P.

21. Rodier J. (2009): L'analyse de l'Eau naturelle, Eau résiduelle, Eau de mer. Dunod techniques 8èmeédition, 1384 p.

22. Piper A.M. (1953). A graphic procedure in the geochemical interpretation of water analysis. US Geol Surv Groundwater Note 12 Sulin VA (1948) Water of petroleum formation in systems of natural waters. Costoptekhizdat, Moscow, 96 pp

23. Soro N. (2002) : Hydrochimie et géochimie isotopique des eaux souterraines du degré carré de Grand-Lahou et ses environs (sud-ouest de la Côte d'Ivoire). Implication hydrologique et hydrogéologique. 
Thèse deDoctorat d'État ès Sciences Nat. Univ. Cocody Abidjan. 272p.

24. Soro N., Ouattara L., Dongo K., Kouadio E. K., Ahoussi E. K., Soro G., Oga M. S., Savane I. Biemi J. (2010) .Déchets municipaux dans le District d'Abidjan en Côte d'Ivoire: sources potentielles de pollution des eaux souterraines, Int. J. Biol. Chem. Sci. 4(6): 2203-2219.

25. Youan Ta M, Lasme OD, Baka D, Lasm T, Jourda PJ, Biemi J. (2015). Analyse des propriétés hydrodynamiques de l'aquifère fissuré du socle paléoprotérozoïque: Aide à l'approvisionnement en eau potable des populations de la région de Bondoukou (Nord-est de la Côte d'Ivoire).13(3): 563-564. 